CHD in Hainan Li and Han nationality. The higher TG level and the lower HDL-C level may be the risk factor in Hainan Li and Han nationality.

\section{e0399 THE ACE GENE POLYMORPHISMS DISTRIBUTION STATUS IN HAINAN LI AND HAN PEOPLES WITH CORONARY HEART DISEASE}

doi:10.1136/hrt.2010.208967.399

${ }^{1}$ Yun Meiling, ${ }^{2}$ Zhong Janghua, ${ }^{3}$ Zheng Yin, ${ }^{2}$ Zeng Yu, ${ }^{2}$ Li Tianfa, ${ }^{4}$ Zhang Yong. ${ }^{1}$ Affiliated Hospital; Hainan Medical College; ${ }^{2}$ Affiliated Hospital Hainan Medical College; ${ }^{3}$ Hainan Provincial Peoples Hospital; ${ }^{4}$ Hainan Province Diseases of The Aged Hospital

Objective To explore the significance of the ACE gene insertion/ deletion (I/D) polymorphism in peoples of Hainan $\mathrm{Li}$ and $\mathrm{Han}$ nationality with coronary heart disease (CHD).

Methods Used the PCR to detect the polymorphisms of ACE gene insertion/deletion (I/D) in 150 patients with CHD and 150 healthy people from Hainan Han and Li nationality respectively. Observed the genotype frequencies and allele frequencies of DD, DI and II. Specific PCR detection was performed for patients who have been determined as DD by normal PCR to reduce misclassification rate. Meanwhile detected the blood lipid, the lipoprotein, the blood pressure, the blood sugar in all people. Used the multiple regression analysis to find out the risk factor in CHD patients.

Results The genotype frequencies of DD in the CHD group of Han and Li nationality are significantly higher than the control group of $\mathrm{Han}$ and $\mathrm{Li}(\mathrm{p}<0.05)$. There were no significant differences in the genotype frequencies of DD, DI and II between the Han and Li with CHD. By the multiple regression analysis it shows: the genotype frequencies of DD in the CHD group of Han and Li nationality increased, the high density lipoprotein cholesterol (HDL-C) level in the CHD group of Han and Li nationality decreased. The triglyceride (TG) level in the CHD group of Han nationality increased.

Conclusions The genotype frequencies of DD are associated with CHD. The susceptibility of CHD in Han and Li nationality is the same. Increasing the HDL-C level can protect the CHD patients. The high level of TG is the independent risk factor in Hainan Han people with CHD.

\section{e0400 MOBILISE AUTOLOGOUS BONE MARROW STEM CELLS TO REPAIR INFARCTED MYOCARDIUM}

doi:10.1136/hrt.2010.208967.400

Bo Wang, Xiaojuan Bai. The First Affiliated hospital of China Medical University

Objective Autologous bone marrow stem cells were mobilised and released by cytokines. On the basis of homing and injured microenvironment theories, we investigated the effective reparation of situ transplantation for acute myocardial infarction in rats.

Methods (1) We divided 60 Wistar rats into situ transplantation groug and control group; To duplicate rat' acute myocardial infarction model by injection of drug; CK, LDH level were checked by automatic biochemistry analyser; (2) 30 Wistar rats were injected rhG-CSF $50 \mu \mathrm{g} / \mathrm{kg}$.day; (3) We did a control analysis on pathological section between two groups by histological staining technique and computer graphic analysis; (4) Heart function were checked by polygraph system after AMI model 4 weeks.

Results (1) Serum cardiac enzymes were higher after AMI model $48 \mathrm{~h}$ than it before AMI model. We saw local necrosis region with grid and trabs shape in endocardium of left ventricular apex and papillary muscle; (2) The infarction size in situ transplantation group was smaller than one in control group; (3) The heart function parameters improved significantly in situ transplantation group compared with control group.

Conclusions (1) Rat's acute myocardial infarction model could be completed by injecting $10 \mathrm{mg} / \mathrm{kg}$ isoprenaline interaperitoneally; (2) Infarcted myocardium were repaired and heart function improved by using cytokines.

\section{E0401 CHANGES OF INTRACELLULAR CALCIUM CONCENTRATION IN CARDIAC-LIKE MYOCYTES}

doi:10.1136/hrt.2010.208967.401

Wang bo, Bai xiaojuan. The First Affiliated hospital of China Medical University

Objective To study the effects of verapamil, endothelin on $\left[\mathrm{Ca}^{2+}\right]_{\mathrm{i}}$ in cardiac-like myocytes derived of bone marrow mesenchymal stem cells.

Methods (1) Bone marrow mesenchymal stem cells and cardiac myocytes were cultured by primary method; (2) Bone marrow mesenchymal stem cells differentiated into cardiac-like myocytes by 5-azacytidine induction agent; (3) We divide our test into three groups, including the first generation bone marrow mesenchymal stem cells, cardiac-like myocytes and cardiac myocytes; (4) The alteration of $\left[\mathrm{Ca}^{2+}\right]_{\mathrm{i}}$ affected by verapamil, endothelins was observed with $\left[\mathrm{Ca}^{2+}\right]_{\mathrm{i}}$ fluorescence imagine system in bone marrow mesenchymal stem cells, cardiac-like myocytes and cardiac myocytes.

Results (1) Cardiac myocytes were set up through trypsogen digestion method; (2) The alteration of $\left[\mathrm{Ca}^{2+}\right]_{\mathrm{i}}$ affected by verapamil was observed with $\left[\mathrm{Ca}^{2+}\right]_{\mathrm{i}}$ fluorescence imagine system. There was a same trend of variability between cardiac-like myocytes and cardiac myocytes, but no changes in bone marrow mesenchymal stem cells; (3) The alteration of $\left[\mathrm{Ca}^{2+}\right]_{\mathrm{i}}$ affected by endothelin was observed with $\left[\mathrm{Ca}^{2+}\right]_{\mathrm{i}}$ fluorescence imagine system. There was a same trend of variability that fluorescence intensity gradually strengthened with intervention time extended.

Conclusions After affected by verapamil and endothelins, there was a same trend of $\left[\mathrm{Ca}^{2+}\right]_{\mathrm{i}}$ changes between cardiac-like myocytes from bone marrow mesenchymal stem cells and cardiac myocytes. It shows that some common electrophysiological characteristics exist in cardiac-like myocytes and cardiaomyocytes.

\section{e0402 THE EXPRESSION AND RELATION OF THE VITAMIN D3 UP. REGULATED PROTEIN 1 IN PERIPHERAL BLOOD MONONUCLEAR CELLS FROM PATIENTS WITH CORONARY ARTERY DISEASE}

doi:10.1136/hrt.2010.208967.402

Shui Xing, Wu Zhen. The Third Affiliated Hospital of Sun Yat-sen University

Aims Vitamin D3 up-regulated protein 1(VDUP1) is a stressresponse gene and participates in oxidative stress, inflammation, apoptosis, proliferation, glucose homeostasis and lipid metabolism. All of these biological effects play important roles in atherosclerosis. Hence, we made an attempt to study the gene expression of VDUP1 using PBMCs from patients with coronary artery disease (CAD).

Methods The total RNA of PBMCs were acquired from 20 normal persons without history of cardiovascular disease and 72 patients with $\mathrm{CAD}$. The $\mathrm{CAD}$ group was divided into 6 subgroups judged by following risk factor. The subgroup 1 was patient without hyperlipidaemia, hypertension and diabetes. The subgroup 2 was patient with hypertension only. The subgroup 3 was patient with hyperlipidaemia only. The subgroup 4 was patient with diabetes only. The subgroup 5 was patient with hyperlipidaemia plus hypertension. 
The subgroup 6 was patient with diabetes plus hypertension. The expression of mRNA level were identified by Real-time RT-PCR.

Results The ratios of VDUP1/ $\beta$-Actin of two groups were skewed distribution. In $\mathrm{CAD}$ group, the maximum was 630.346, the minimum was 1.000 , the median was 5.205. In control group, the maximum was 837.532 , the minimum was 2.395 , the median was 80.449. By logarithmic transformation, the results indicated the expression of VDUP1 in PBMCs from patients with CAD were markedly down-regulated than that from control group $(p<0.05)$. The expression of VDUP1 in PBMCs from patients with single risk factor were down-regulated than that from patients with multiple risk factors in $\mathrm{CAD}$ group $\left(\mathrm{P}_{2}=0.044, \mathrm{P}_{4}=0.033\right)$.

Conclusion These findings shed new light onto the mechanisms of $\mathrm{CAD}$, demonstrate that the expression of VDUP1 in PBMCs from treated patients with $\mathrm{CAD}$ has a negative correlation to $\mathrm{CAD}$, and sugguest that modulating the function of VDUP1 may open a new era of the therapy for $\mathrm{CAD}$.

\section{E0403 THE DIAGNOSIS OF CORONARY ARTERY ORIGIN ANOMALIES WITH DUAL-SOURCE CT AND ITS CLINICAL SIGNIFICANCE}

doi:10.1136/hrt.2010.208967.403

Li Dongye, Zhang Yonggeng, Xu Kai, Long Bin, Xie Lixiang, Xia Yong, Zhang Yanbin. The Affiliated Hospital of Xuzhou Medical College

Purpose To investigate the clinical value of dual-source computer tomography (DSCT) in detecting anomalous origin of coronary artery for adult patients.

Materials and methods A retrospective evaluation to identify 3903 patients who underwent DSCT coronary angiography from Jan 2009 to Jan 2010.

Results All images were considered to be suitable for evaluation. Forty-two of 3903 patients were detected to have coronary atrery origin anomaly. The incidence is $1.08 \%$. They include 26 cases with an anomalous origin of right coronary artery $(0.67 \%), 13$ cases with an anomalous origin of left coronary artery $(0.33 \%), 3$ cases with single coronary artrey $(0.08 \%)$.

Conclusion DSCT coronary angiography that provide accurate depiction of anomalous coronary origin and course along with the complex anatomical relation with the adjacent structures can be considered as a first-line imaging method for delineating coronary arterial anomalies.

\section{E0404 RELATIONSHIP BETWEEN INSULIN RESISTANCE AND}

doi:10.1136/hrt.2010.208967.404

${ }^{1}$ Wei Ren-long, ${ }^{1}$ Hasimu Buaijiaer, ${ }^{1}$ Yuan Bin-bin, ${ }^{2}$ Yang Zhi-jian. ${ }^{1}$ Department of Cardiology, Nanjing Benq Hospital, The Affiliated Hospital of Nanjing Medical University; ${ }^{2} 2$ department of Cardiology, The First Affiliated Hospital of Nanjing Medical University

Objective To determine insulin resistance in patients with coronary heart disease and explore the relationship between insulin resistance and coronary atherosclerosis, cardiovascular risk factors.

Methods The study population consisted of 506 consecutive patients (376 male and 130 female) who underwent coronary angiography and laboratory measurements for suspected or known coronary heart disease. The severity of coronary atherosclerosis was defined by using Gensini's score system. High specific BA-ELISA assays for true insulin was used. Insulin resistance was assessed by HOMA index. 506 cases were allocated into four groups according to HOMA index. Analysis of variance, kruskal-wallis test and $\chi^{2}$ test was employed to investigate the distribution of the clinical data in four groups according to HOMA index. Spearman's correlation analysis and multivariate stepwise linear regression analysis were employed to explore the relationship between HOMA index and Gensini's score, the cardiovascular risk factors.

Result One-way ANOVA and kruskal-wallis test indicated that age, triglyceride, apolipoprotein A, high density lipoprotein cholesterol, uric acid, BMI and Gensini's score differed among four groups according to HOMA index $(p<0.05)$. Spearman's correlation analysis suggested that HOMA index was positively correlated with triglyceride, apolipoprotein $\mathrm{B}$, uric acid, BMI and Gensini score but HOMA index was negatively correlated with apolipoprotein $\mathrm{A}$ and high density lipoprotein cholesterol. Multivariate stepwise linear regression analysis showed that BMI had the independent association with HOMA index ( $\mathrm{r}=0.090, \mathrm{p}=0.05)$.

Conclusion Insulin resistance existed in the patients with coronary heart disease. Insulin resistance was positively correlated with coronary atherosclerosis and was independently correlated with $\mathrm{BMI}$ in the patients with coronary heart disease.

\section{e0405 EFFECT OF TELMISARTAN ON CARDIAC FUNCTION AND BRAIN NATRIURETIC PEPTIDE IN PATIENTS WITH CHRONIC HEART FAILURE}

doi:10.1136/hrt.2010.208967.405

Bin Hu, Yujie Zhou, Dongmei Shi, Yingxin Zhao. Beijing Anzhen Hospital

Objective To evaluate telmasartan on cardiac function and brain natriuretic peptide (BNP) patients with chronic heart failure (CHF). Methods We enrolled 120 patients with CHF, NYHAI-III, age 30-79 (61.25 \pm 10.18$)$ years. All the patients were randomly assigned to 2 groups: standard therapy group $(n=60$, receiving ACEI, digoxin, diuretic, $\beta$-blcoks), telmasartan treatment group ( $n=60$ receiving telmasartan in addition to the standard therapy). These patients were treated for 1 years, and plasma levels of BNP and left ventiricular ejection fraction (LVEF) were measured before and after treartments.

Results No significant differences in baseline characteristics were observed between the two groups. After treatment, BNP plasma levels both decreased and LVEF increased significantly in two groups. BNP plasma levels in telmasartan treatment group were lower than that in standard group and LVEF higher at 1 year follow-up.

Conclusion Telmasartan in addition to the standard therapy can improve the cardiac function and desease BNP plasma levels.

\section{e0406 HIGH SENSITIVITY C-REACTIVE PROTEIN AND THE RISK OF STENT THROMBOSIS AND CARDIOVASCULAR EVENTS}

doi:10.1136/hrt.2010.208967.406

Bin Hu, Yujie Zhou, Yuyang Liu, Dongmei Shi, Yingxin Zhao. Department of Cardiology, Beijing Anzhen Hospital, Capital Medical University, Beijing, China

Background C-reactive protein (CRP) is one of the acute phase proteins that increase during systemic inflammation. It's been suggested that testing CRP levels in the blood may be an additional way to assess cardiovascular disease risk. A more sensitive CRP test, called a highly sensitive C-reactive protein (hs-CRP) assay, is available to determine heart disease risk. However, and the association between CRP and stent thrombosis after drug-eluting stent implantation has not been defined.

Objective To investigate in a follow-up study whether high-sensitivity C-reactive protein (hs-CRP) predicts coronary heart disease (CHD) events and stent thrombosis in subjects undergone drugeluting stent implantation. 